\title{
ПАПИЛЛЯРНЫЙ РАК ЩИТОВИДНОЙ ЖЕЛЕЗЫ У ПАЦИЕНТА С СЕМЕЙНЫМ АДЕНОМАТОЗНЫМ ПОЛИПОЗОМ
}

\author{
Авторы: А.В. Болмасова, М.А. Меликян, А.Р. Савкина, М.А. Курникова, С.Г. Подлипаева,
} Н.В. Бабаскина, В.А. Петеркова

ФГБУ «НМИЦ Эндокринологии» Минздрава России, Москва

ФГБУ «НМИЦДГОИ им. Дмитрия Рогачева» Минздрава России, Москва

ВВЕДЕНИЕ: Семейный аденоматозный полипоз (синдром Гарднера, FAP-1 ОМIM\# 175100) это редкое аутосомно-доминантное заболевание (частота 1: 14 025), характеризующееся наличием множественных полипов в толстой кишке в сочетании с экстраколониальными опухолями, среди которых выделяют остеомы черепа, эпидермоидные кисты, фибром, десмоидные опухоли, рак щитовидной железы, злокачественные образования печени и почек. Заболевание связано с доминантыми мутациями в гене АРС локализованном на длинном плече 5 хромосомы (5q21).

ОПИСАНИЕ СЛУЧАЯ: Пациентка 16 лет, родилась в срок от 2 беременности (1 - выкидыш), с массой при рождении: 4100 г, длиной тела 51 см. Наследственный анамнез отягощен по материнской линии (у матери-рак прямой кишки, погибла в возрасте 25 лет).

С 11 лет наблюдалась у невролога с диагнозом: Юношеская абсансная эпилепсия, получала противосудорожную терапию. С 14 лет у девочки отмечалась железодефицитная анемия рефрактерная к терапии пероральными препаратами железа. В 15 лет при плановом обследовании выявлено узловое образование левой доли щитовидной железы размером 13,6×8,5×10,5 мм, смешанной структуры за счет анэхогенного компонента. По результатам тонкоугольной аспирационной биопсии (ТАБ) — коллоидный зоб (Bethesda II). При динамическом обследовании отмечался рост образования, при проведении ультразвукового исследования щитовидной железы в 16 лет выявлен левосторонний узловой зоб размером 16×13×10 мм (EU-TIRADS 4), по данным ТАБ - диагностическая категория Bethesda V. B гормональном профиле-эутиреоз, кальцитонин в пределах нормы. Учитывая сохраняющуюся железодефицитную анемию средней степени тяжести (гемоглобин 88г/л), отягощённый семейный анамнез, заподозрено кишечное кровотечение. Ребенку был проведен анализ кала на скрытую кровь, который показал положительный результат. Проводилась терапия парентеральными формами препаратов железа и рекомбинантным эритропоэтином с положительным эффектом.

На основании клинико-лабораторной картины заподозрен Семейный аденоматозный полипоз. Проведено молекулярно-генетическое исследование: в гене АРС, в интроне 11 выявлен гетерозиготный вариант c.1408+1 delG, не имеющий популяционных частот и не описанный в литературе.

Пациентке была проведена колоноскопия, по результатам которой выявлены множественные (более 100) полиповидные образования толстой кишки. Была проведена полипэктомия наиболее крупных полипов, по результатам гистологического исследования - аденоматозный полипоз кишечника.

В дальнейшем ребенку выполнена левосторонняя гемитиреоидэктомия с истмусэктомией. По результатам гистологического исследования был подтвержден диагноз папиллярного рака щитовидной железы (крибриформно-модулярный вариант).

Таким образом, учитывая наличие отягощенного семейного анамнеза, папиллярного рака щитовидной железы в сочетании с аденоматозным полипозом кишечника, а также на основании результатов молекулярно-генетического исследования пациентке был установлен диагноз: Семейный аденоматозный полипоз (синдром Гарднера).

Учитывая высокий риск развития рака толстой кишки, планируется проведение колопрокэктомии с формированием тонкокишечного резервуара и илеоанального анастомоза, показано регулярное УЗИ щитовидной железы, скрининг на иные составляющие синдрома.

Выводы: пациентам с раком щитовидной железы, при наличии отягощенного семейного анамнеза в отношении колоректального рака необходимо проведение дополнительного обследования для исключения Семейного аденоматозного полипоза.

КЛЮЧЕВЫЕ СЛОВА: СИндром Гарднера, семейный аденоматозный полипоз, папиллярный рак щитовидной железы 